\title{
Mechanism of Sini Decoction on Coronary Heart Disease in Molecular Level
}

\author{
Weiye Tao \\ College of Medical Information \\ Guangdong Pharmaceutical University \\ Guangzhou, China \\ taoweiye@163.com
}

\author{
Laiyou Wang \\ Institute of Chinese Medicinal Sciences \\ Guangdong Pharmaceutical University \\ Guangzhou, China \\ wly1999@sina.com
}

\author{
Guohua Cheng, Jun Liu, Langping \\ Tang \\ College of Medical Information \\ Guangdong Pharmaceutical University \\ Guangzhou, China \\ langping_tang@sina.com
}

\begin{abstract}
Sini Decoction is a traditional Chinese medicine which has a curative effect. The mode of action between small molecules and the targets were presented visually, which provided an in-depth interpretation about the pharmacodynamic material basis. It is valuable for the research and development of new drugs. Experimental results show that we can reveal the treatment mechanism of Sini Decoction in molecular level by molecular docking.
\end{abstract}

Keywords-computational simulation; molecular docking; Sini Decoction; computer-aided drug design

\section{INTRODUCTION}

Sini Decoction is constituted with FuZi (radix aconiti lateralis preparata), GanJiang (rhizoma zingiteris) and GanCao (radix glycyrrhizae). From the treatise of Zhang Zhongjing's Shang Han Lun (Treatise on Febrile Disease), we can know it can treat coronary heart disease. Liu [1] analyzed the clinical symptoms of 49 coronary heart disease patients; the medicine all of these patients took was Sini Decoction. By observation on clinic comparison, the effective rates were $92 \%$ in the treatment group and $87 \%$ in the control group. ECG changes: in treatment group of 49 cases, 18 cases were significantly effective, 23 cases effective, 7 cases had no effect, and one case got heavier. When come to control group, the total effective rate was only $75.3 \%$. Liang [2] had observed clinical effect of Sini decoction on stenocardia for coronary heart disease. 65 cases of stenocardia for CHD with deficiency of yang or cold syndrome were randomly divided into Sini decoction therapeutic group and isosorbide dinitrate controll group. Drug effects of the two groups were compared by clinical symptoms, electrocardiogram (ECG), myocardial oxygen consumption and cardiac function. Results compared with isosorbide dinitrate showed that Sini decoction had better effects on reducing myocardial oxygen consumption and improving cardiac function. It was similar to improving clinical symptoms and decreasing frequency of stenocardia and on stenocardia for coronary heart disease. The conclusion was that Sini decoction can treat stenocardia for CHD with deficiency of yang or cold syndrome.

Traditional Chinese medicine is a complex system. It is difficult to find out the pharmacodynamic material basis and the mechanism. The reason why Chinese medicine is not understood in the Western world is that the means and methods of Western medicine can't apply to traditional Chinese medicine very easily. Molecular docking can reveal the pharmacodynamic material basis of Chinese medicine. It can help Western world understand Chinese medicine and guide rational drug design. Based on computational chemistry, we conducted computational simulation of Sini Decoction in treating coronary heart disease to reveal the mode of action between small molecules and the targets associated with coronary heart disease.

\section{MATERIALS AND METHODS}

\section{A. Virtual screening.}

We can predict the activity of the chemical constituents with virtual screening techniques based on computational chemistry. After virtual screening, we can do pharmacological experiments to test and verify the ingredients' real activity based on the prediction result. The basic idea of virtual screening is: for a large number of compounds which structure is known, we use a computer to simulate its interaction with a given target. If the computer has theoretically proved activity of these compounds for the target, then we can obtain these compounds to conduct the corresponding pharmacological experiments to validate the theoretical activity. So we can see that this can quickly filter out the real active ingredient Virtual screening is realized through computer software, the theory and software is summed up on the basis of experimental or clinical data on drugs interaction with the human body, so it tends to have a relatively high accuracy and can guide rational drug design[3].

\section{B. Software required in experiment.}

In computational simulation, the software used was Autodock vina [4], Pymol [5] and Cytoscape [6]. AutoDock vina is a suite of automated docking tools used for predicting the interaction of ligands with biomacromolecular targets. PyMOL is a user-sponsored molecular visualization system on an open-source foundation. Cytoscape is an open source bioinformatics 
software platform for visualizing molecular interaction networks and biological pathways.

\section{Network construction and visualization of interaction between molecules and targets}

We constructed each Chinese herbal's drug - target network diagram using the software Cytoscape. Virtual screening can find out which drug molecules of Chinese herbal medicine may have efficacy, but the mode of action between the drug molecules and targets are not clear. Therefore, it is necessary to analyze the mode of action between drug molecules and targets, which provides an indepth interpretation about the pharmacodynamic material basis, and provide a high reference value for the research and development of new drugs.

In previous work, we found out the pharmacodynamic material basis of Sini Decoction through virtual screening using the software of Autodock. In this experiment, we used Autodock Vina to conduct virtual screening. We collected 146 compounds in Sini Decoction from Traditional Chinese Medicine Database@Taiwan [7]. The targets related to coronary heart disease were from Therapeutic Target database [8].

According to the results, we observed a few of ligandtarget complexes, and analyzed. The mode of action between small molecules and the targets associated with coronary heart disease.

\section{RESUlts AND DiscUSSIONS}

The docking results of 146 small molecule compounds and the corresponding targets are shown in Table 1. In order to conduct network construction and analysis, we should import the small molecules and drug targets into Cytoscape. Fig.1, Fig.2 and Fig.3 describe the interaction network between drug and the target in form of visualization. In these figures, square boxes represent targets, small molecule compounds are represented by round dots. As can be seen from Table 1 and the three figures, there are interactions between a total of 99 small molecules and 11 targets.

Figure 1 depicts the drug - target network of FuZi, we can know that drug molecules in FuZi can act on 9 targets, and 1ZD5 is one of the most important target proteins. Figure 2 depicts the drug - target network of GanJiang, we can know that drug molecules in GanJiang can act on 8 targets, and 1ZD5 is also one of the most important target proteins. Figure 3 depicts the drug - target network of GanCao, we can know that drug molecules in GanJiang can act on 11 targets, and 1ZD5, 1TY7, 1FjS, ACE are the important targets protein.

In the development of many cardiovascular diseases, such as coronary heart disease and pulmonary embolism, the formation of intravascular clot plays an important role. Factor $\mathrm{Xa}$ is a serine protease. It can activate thrombin (factor IIa) and plays a key regulatory role in the bloodcoagulation cascade. Therefore, factor $\mathrm{Xa}$ is an important target for the design of anti-thrombotics [9]. There is much interest in the development of factor Xa inhibitors for the intervention on thrombic diseases.

In Chinese traditional medicine, FuZi is one of the most important Chinese medicinal material. Delphin and Demethyldelavaine A are two pure compounds isolated from FuZi, so we choose them as the drug molecules to analyze the pharmacological action.

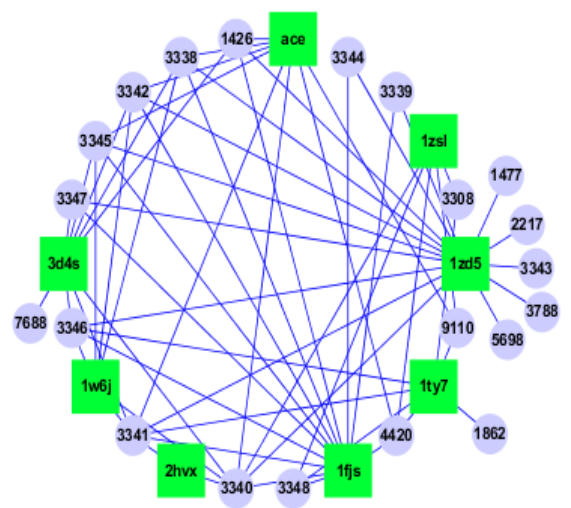

Figure 1.Drug-target network of FuZi

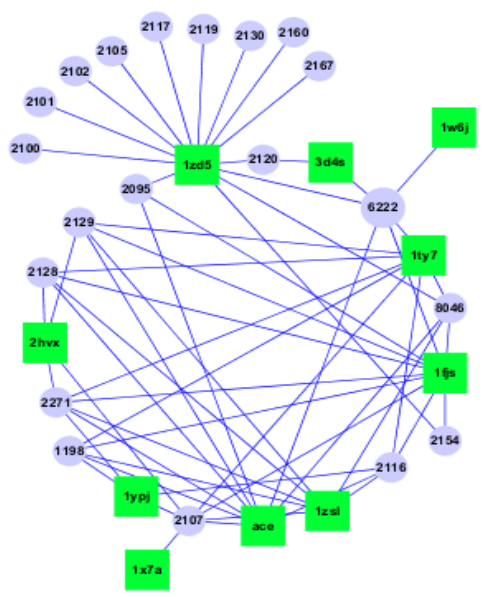

Figure 2.Drug-target network of GanJiang

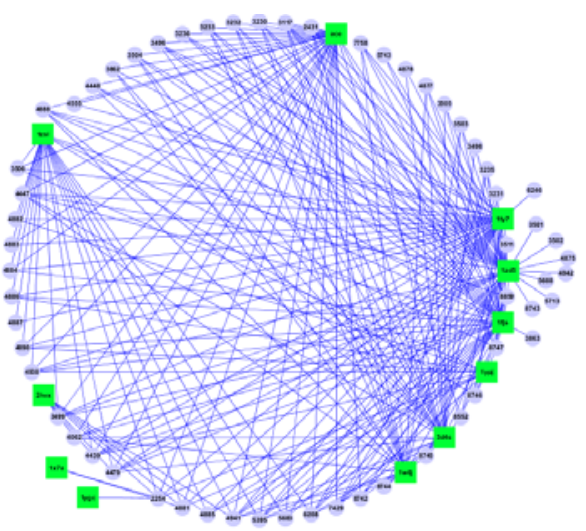

Figure 3.Drug-target network of GanCao 
Because of the importance of the target factor Xa, we choose the factor Xa (PDB ID is 1FJS) as the example to analyze the interaction between drug and targets.

Figure 4 shows the interaction between delphin and 1FJS. We can see the ligand-receptor complexes obtained by molecular docking. In the binding site, one stable hydrogen bond interaction has been formed with residues of HIS-57, SER-214 and ARG-222 separately.

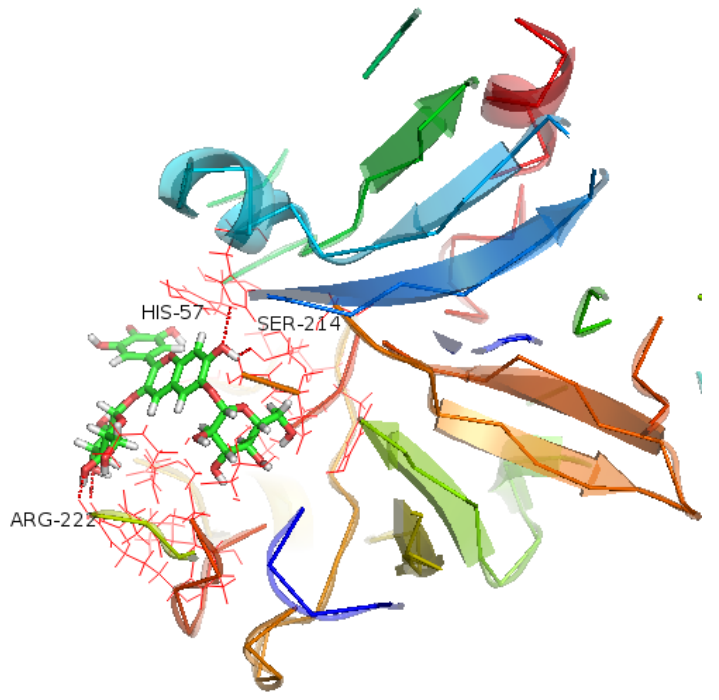

Figure 4. Interaction between Delphin and 1FJS

Figure 5 shows the interaction between 1FJS and Demethyldelavaine A. In the binding site, one stable hydrogen bond interaction has been formed with residues of SER-214 and TYR-228 separately. We can see that SER244 is very important in the ligand-target complex.

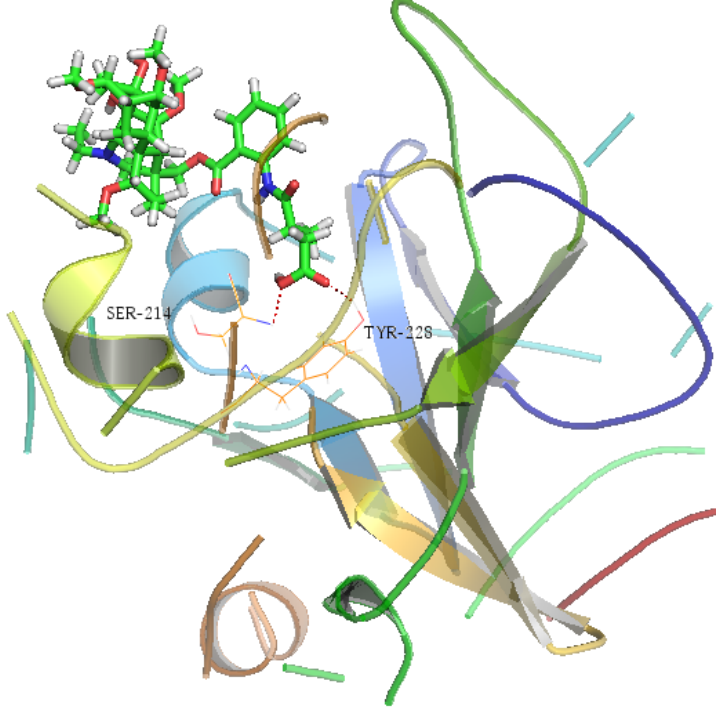

\section{CONCLUSIONS}

Through computational simulation of Sini Decoction on coronary heart disease, we can reveal the mode of action between small molecules and the targets associated with coronary heart disease.

Traditional Chinese medicine (TCM) holds an important role in medical diagnosis and treatments in China for thousands of years, but TCM is less recognized in the Western world. With the development of computational technologies, computer-aided drug design has been greatly accelerated. Recently, pharmaceutical companies have increasingly paid their attentions toward traditional Chinese medicine (TCM) for novel lead compounds [10].

In modern medicine view, Sini Decoction and its addition and subtraction can treat many other diseases, including pulmonary heart disease, myocardial disease, sinus bradycardia , ischemic stroke, thrombotic occlusion vasculitis, dysmenorrhea, schizophrenia, headache, insomnia, newborn scleroderma and prostatic hyperplasia. Finding out the pharmacodynamic material basis and treatment mechanism of Sini Decoction for above diseases is also very important.

\section{REFERENCES}

[1] Hong Liu, "Efficacy of Chinese medicine of Sini Decoction on coronary heart disease", Chinese Medical Journal of Metallurgical Industry, vol. 23(2), pp. 258-259, 2006.

[2] Yingming Liang," Clinical Study on Sini Decoction on Treating Stenocardia for Coronary Heart Disease", Journal of Chinese Medicinal Materials, vol. 28(8),pp. 737-738, 2005.

[3] Wenyu Yang, Deguang Wan, Xinyu Yang, "Thinking and primary practice on discovery of pharmacodynamic material basis of Chinese materia medica assisted by virtual screening method", Chinese Traditional and Herbal Drugs, vol. 42(9), pp.1665-1672, 2011.

[4] O. Trott, A. J. Olson, "AutoDock Vina: improving the speed and accuracy of docking with a new scoring function, efficient optimization and multithreading", Journal of Computational Chemistry, vol. 31, pp.455-461, 2010.

[5] The PyMOL Molecular Graphics System, Version 1.3, LLC.

[6] Smoot ME, Ono K, Ruscheinski J, Wang PL, Ideker T, "Cytoscape 2.8: new features for data integration and network visualization", Bioinformatics, vol. 27(3), pp. 431- 432, 2011.

[7] Chen CY-C, "TCM Database@Taiwan: The World's Largest Traditional Chinese Medicine Database for Drug Screening In Silico", PLoS ONE, vol. 6(1): e15939, 2011.

[8] Chen X, Ji ZL, Chen YZ, "TTD: therapeutic target database", Nucleic Acids Res., vol. 30(1), pp. 412-415, 2002.

[9] Whitlow, M., Arnaiz, D.O., Buckman, B.O. et al , "Crystallographic analysis of potent and selective factor Xa inhibitors complexed to bovine trypsin", Acta Crystallogr D Biol Crystallogr, vol. 55, pp. 1395-1404, 1999.

[10] Katharine Sanderson, "Databases aim to bridge the East-West divide of drug discovery", Nature Medicine, vol. 17(12), pp. 1531, 2011. 
Table 1 NUMBERS OF EFFECTIVE MOLECULE IN FUZI, GANJIANG AND GANCAO

\begin{tabular}{|l|l|l|l|l|}
\hline Target Name & $\begin{array}{l}\text { PDB } \\
\text { ID }\end{array}$ & $\begin{array}{l}\text { Molecules } \\
\text { in FuZi }\end{array}$ & $\begin{array}{l}\text { Molecules } \\
\text { in GanJiang }\end{array}$ & $\begin{array}{l}\text { Molecules in } \\
\text { GanCao }\end{array}$ \\
\hline Angiotensin-converting enzyme & 1UZE & 9 & 8 & 33 \\
\hline Beta-2 adrenergic receptor & 3D4S & 2 & 9 & 28 \\
\hline Soluble epoxide hydrolase & 1ZD5 & 15 & 15 & 42 \\
\hline mRNA of Factor XI & 1ZSL & 7 & 5 & 22 \\
\hline Coagulation factor IX & 1X7A & 1 & 0 & 2 \\
\hline Coagulation factor VII & 1YGC & 0 & 0 & 1 \\
\hline Coagulation factor Xa & 1FJS & 0 & 12 & 40 \\
\hline Glycoprotein IIb/IIIa receptor & 1TY7 & 8 & 7 & 32 \\
\hline Thrombin & 1YPJ & 4 & 0 & 12 \\
\hline Bile acid receptor & 1OSH & 0 & 0 & 0 \\
\hline Lanosterol synthase & 1W6J & 1 & 6 & 20 \\
\hline Chymase & 2HVX & 4 & 2 & 7 \\
\hline
\end{tabular}

